\title{
Matched filter based algorithm for blind recognition of OFDM systems
}

\author{
Abdelaziz Bouzegzi*, Philippe Ciblat ${ }^{\dagger}$, and Pierre Jallon* \\ ${ }^{*}$ CEA-LETI, MINATEC - Grenoble, France (Email: abdelaziz.bouzegzi@cea.fr, pierre.jallon@cea.fr) \\ ${ }^{\dagger}$ ENST, Paris, France (Email: philippe.ciblat@enst.fr)
}

\begin{abstract}
In the Cognitive Radio context, sensing process is a crucial task. The cognitive devise has to be able to detect and to identify several radio systems. As more standards are now based on OFDM modulation the paper aims with the parameters estimation of such a modulation. All actual OFDMbased standards differ from their subcarrier spacing therefore it will be ingenious to focus on this parameter to identify these systems in a non data aided context. We propose a new efficient algorithm based on the matched filter principle. The behavior of the proposed approach will be studied in the context of practical impairments like frequency and/or time offsets and a multipaths fading channel. Finally, the performance of the proposed algorithm will be evaluated in contrast with the state of art methods by means of computer simulations.
\end{abstract}

\section{INTRODUCTION}

The cognitive radio concept has been first introduced by [1] and consists for a radio in carry out additional functionalities to adapt its transmission parameters in regard of its spectral environnement. Consequently, a cognitive terminal needs to be able to detect the access points of different systems in it neighbourhood and to recognize their used standards.

The Opportunistic Radio is one of the multiple Cognitive Radio applications. It aims that the opportunistic device has to be able to detect the unused spectrum bands and to adapt its transmission parameters in order to transmit within these bands. Indeed, determining the frequency band used by each system can not be considered as an efficient identification tool and thus new blind techniques seem to be needful.

Most of popular standards use OFDM modulations (e.g. WiFi, WiMax, DVB, 3GPP/LTE). Nevertheless, their intercarrier spacing parameters enable to distinguish them from each others. In fact, the intercarrier spacing is equal to $15.625 \mathrm{kHz}$, $10.94 \mathrm{kHz}, 312.5 \mathrm{kHz}, 1.116 \mathrm{kHz}, 15 \mathrm{kHz}$ for Fixed WiMAX [2], Mobile WiMAX [2], WiFi [3], DVB-T [4], 3GPP/LTE [5] respectively. The problem of the system recognition issue hence boils down to the estimation of the intercarrier spacing of the receive signal. We focus on this problem in this paper. Obviously, the proposed estimation algorithm also applies to military contexts.

Only few results about this problem can be found in the literature, mainly, in [6], [7], [8] which are all based on the correlation property of the cyclic-prefixed OFDM signals. In fact, the cyclic prefix is added on each OFDM symbol by copying a part of the useful part at the beginning of the symbol. OFDM signals hence exhibit a correlation peak at a time lag equal to their useful time. As the useful time is also equal to the inverse of the intercarrier spacing, the system recognition can be done accordingly. Nevertheless, it is straightforward to understand that if the cyclic prefix is short (in regard to the useful time of the OFDM symbol), the correlation peak is low and the correlation based methods fail. In practice, these contexts occur at least for DVB-T signals and WiMax signals for which the cyclic prefix may be very short. Moreover, this approach is sensible to multipaths channels and falls down if another scheme of OFDM signals is used (ex: ZP-OFDM).

In this paper we propose an alternative method based on the matched filter principle. We prove the effectiveness of the proposed cost function and we show its robustness to any scheme of OFDM signals (short CP, ZP-OFDM). Furthermore, we show that this approach resists in a multipaths channel context. The paper is organized as follows: In Section II, we derive the signal model. In Section III we give the guidelines of the proposed method. In Section $\mathrm{V}$ we show how the proposed algorithm can be modified deal with a carrier offset. Finally, Section V is devoted to numerical illustrations where a comparison with the autocorrelation based method is also drawn.

\section{Signal MOdel}

In Additive White Gaussian Noise channel, the OFDM receive signal sampled at a rate $1 / T_{e}$ writes:

$$
\begin{aligned}
y(m) & =\sum_{k=0}^{K-1} \sum_{n=0}^{N-1} \frac{a_{k, n}}{\sqrt{N}} e^{-2 i \pi \frac{n\left(m T_{e}-D T_{c}-k T_{s}\right)}{N T_{c}}} g_{a}\left(m T_{e}-k T_{s}\right) \\
& +b(m)
\end{aligned}
$$

where $K$ is the number of transmit symbols, $N$ is the number of subcarriers and where $1 / T_{c}$ is the information symbol rate in absence of guard interval. To keep all the information of the receive signal, we assume that $T_{e}$ satisfies the Shannon condition, i.e. $T_{e}<T_{c}$. The intercarrier spacing is equal to $1 / N T_{c}$. The length of the cyclic prefix is set to $D T_{c}$. The duration of a whole OFDM symbol is $T_{s}=(N+D) T_{c}$. The sequence $\left\{a_{k, n}\right\}$ represents the transmit unknown data symbols at subcarrier $n$ and OFDM block $k$ and assumed to be independent and identically distributed. The shaping filter $g_{a}(t)$ is assumed to be equal to 1 if $0 \leq t<T_{s}$ and 0 otherwise. The complex-valued noise $b(\mathrm{~m})$ is assumed to be independent and identically distributed, circularly-symmetric 
zero-mean white Gaussian noise. Its variance is equal to $N_{0}$ per real dimension.

The receive samples in Eq. (1) can also be written as the result of a linear transformation. If the vector $\mathbf{y}$ stands for $[y(0), \cdots, y(M-1)]^{\mathrm{T}}$ where $(.)^{\mathrm{T}}$ is the transposition operator and $M$ is the number of the received samples, Eq. (1) indeed rewrites:

$$
\mathbf{y}=\mathbf{F}_{\boldsymbol{\theta}_{\mathbf{0}}} \mathbf{a}+\mathbf{b}
$$

where

- $\mathbf{a}_{k}=\left[a_{k, 0}, \cdots, a_{k, N-1}\right]^{\mathrm{T}}$ of size $N \times 1$

- $\mathbf{a}=\left[\mathbf{a}_{0}^{\mathrm{T}}, \cdots, \mathbf{a}_{K-1}^{\mathrm{T}}\right]^{\mathrm{T}}$ of size $K N \times 1$

- $\mathbf{b}=[b(0), \cdots, b(M-1)]^{\mathrm{T}}$ of size $M \times 1$

In Eq. (2), the matrix $\mathbf{F}_{\boldsymbol{\theta}_{\mathbf{0}}}$ depends on $\boldsymbol{\theta}_{\mathbf{0}}=\left[N, D T_{c}, N T_{c}\right]$. Thanks to the term $g_{a}\left(m T_{e}-k T_{s}\right)$ in Eq. (1), it is straightforward to check that $\mathbf{F}_{\boldsymbol{\theta}_{\mathbf{0}}}$ is defined by block since:

$$
g_{a}\left(m T_{e}-k T_{s}\right) \neq 0 \Leftrightarrow 0 \leq m T_{e}-k T_{s}<T_{s}
$$

which implies that

$$
m \frac{T_{e}}{T_{s}}-1<k \leq m \frac{T_{e}}{T_{s}} .
$$

Consequently, for a given $m$, it exists only an unique value of $k$, denoted by $k_{m}$, such as $g_{a}\left(m T_{e}-k T_{s}\right) \neq 0 . \mathbf{F}_{\boldsymbol{\theta}_{\mathbf{0}}}$ is then composed by null components except the next ones

$$
F_{\boldsymbol{\theta}_{\mathbf{0}}}\left(m, k_{m} N+n\right)=\frac{1}{\sqrt{N}} e^{-2 i \pi n m \frac{T_{e}}{N T_{c}}} e^{2 i \pi n\left(k_{m}+1\right) \frac{D T_{c}}{N T_{c}}}
$$

for $m=0, \ldots, M-1$ and $n=0, \cdots, N-1$.

In the considered context, the terminal just has the knowledge of $\{y(m)\}_{m=0}^{M-1}, M$ and $T_{e}$ and wishes to estimate the value of $N T_{c}$, the inverse of the intercarrier spacing. In this paper, we propose to do that by estimating $\mathbf{F}_{\boldsymbol{\theta}_{\mathbf{0}}}$ which also depends on $N$ and $D T_{c}$.

\section{MATCH FILTER BASED ALGORITHM}

In this section we propose to build an estimator of the unknown parameters based on the match filter approach. The main idea consists of searching the set of parameters $\boldsymbol{\theta}=$ $\left[\widetilde{N}, \widetilde{D T}_{c}, \widetilde{N T}_{c}\right]$ which maximizes the following cost function

$$
J(\boldsymbol{\theta})=\frac{\mathbb{E}\left\{\left\|\mathbf{F}_{\boldsymbol{\theta}}^{\mathrm{H}} \mathbf{y}\right\|^{2}\right\}}{\left\|\mathbf{F}_{\boldsymbol{\theta}} \mathbf{F}_{\boldsymbol{\theta}}^{\mathrm{H}}\right\|_{F}}
$$

where superscript $(.)^{\mathrm{H}}$ stands for the hermitian operator, $\|\mathbf{x}\|^{2}$ is the Euclidian norm of the vector $\mathbf{x}$ and $\|\mathbf{A}\|_{F}$ is the Frobenius norm of the matrix $\mathbf{A}$ equals to $\sqrt{\operatorname{Tr}\left(\mathbf{A}^{\mathrm{H}} \mathbf{A}\right)}$. The effectiveness of the proposed cost function is based on the following theorem:

Theorem 1: In noiseless context, the following equality holds:

$$
J(\boldsymbol{\theta}) \leq J\left(\boldsymbol{\theta}_{\mathbf{0}}\right)=\left\|\mathbf{F}_{\boldsymbol{\theta}_{\mathbf{0}}} \mathbf{F}_{\boldsymbol{\theta}_{\mathbf{0}}}^{\mathrm{H}}\right\|_{F}
$$

and the equality is reached if and only if $\boldsymbol{\theta}=\boldsymbol{\theta}_{0}$.

The proof of Theorem 1 is given using the two following propositions. Let us consider a flat fading channel and a noiseless context. Using the fact that the data symbols are assumed to be i.i.d we note that $\mathbb{E}\left\{\mathbf{a a}^{\mathrm{H}}\right\}=I$ where $I$ is the identity matrix. Therefore, we get $\mathbb{E}\left\{\left\|\mathbf{F}_{\boldsymbol{\theta}}^{\mathrm{H}} \mathbf{y}\right\|^{2}\right\}=\operatorname{Tr}\left(\mathbf{F}_{\boldsymbol{\theta}}^{\mathrm{H}} \mathbf{F}_{\boldsymbol{\theta}_{0}} \mathbf{F}_{\boldsymbol{\theta}_{0}}^{\mathrm{H}} \mathbf{F}_{\boldsymbol{\theta}}\right)$. The first proposition is stated as follows:

Proposition 1: By denoting $A=\mathbf{F}_{\boldsymbol{\theta}} \mathbf{F}_{\boldsymbol{\theta}}^{\mathrm{H}}$ and $B=\mathbf{F}_{\boldsymbol{\theta}_{\mathbf{0}}} \mathbf{F}_{\boldsymbol{\theta}_{\mathbf{0}}}^{\mathrm{H}}$ we get $\operatorname{Tr}\left(A^{\mathrm{H}} B\right) \leq \sqrt{\operatorname{Tr}\left(A A^{\mathrm{H}}\right) \operatorname{Tr}\left(B B^{\mathrm{H}}\right)}$ and the equality holds if and only if $\mathbf{F}_{\boldsymbol{\theta}} \mathbf{F}_{\boldsymbol{\theta}}^{\mathrm{H}}=\mathbf{F}_{\boldsymbol{\theta}_{\mathbf{0}}} \mathbf{F}_{\boldsymbol{\theta}_{\mathrm{o}}}^{\mathrm{H}}$

Proof: The inequality of Proposition 1 can be rewritten as

$$
\left\|A^{\mathrm{H}} B\right\|_{F}^{2} \leq\left\|A^{\mathrm{H}} A\right\|_{F}\left\|B^{\mathrm{H}} B\right\|_{F}
$$

To prove this inequality, an explicit form of $\left\|A^{\mathrm{H}} B\right\|_{F}^{2}$ is given. Using the definition of the Frobenius norm, this term can be expressed as following

$$
\begin{aligned}
\left\|A^{\mathrm{H}} B\right\|_{F}^{2} & =\sum_{i=1}^{\tilde{K} \tilde{N}} \sum_{j=1}^{K N}\left|\sum_{l=1}^{M}\left(A^{\mathrm{H}}\right)_{i l} B_{l j}\right|^{2} \\
& =\sum_{l=1}^{M} \sum_{l^{\prime}=1}^{M}\left[\sum_{i=1}^{\tilde{K} \tilde{N}} A^{*}{ }_{l i} A_{l^{\prime} i}\right]\left[\sum_{j=1}^{K N} B_{l j} B^{*}{ }_{l^{\prime} j}\right]
\end{aligned}
$$

where (.)* stands for the complex conjugate and $\tilde{K}$ is the estimated number of OFDM symbols within the receive signal duration, it is given by $\left\lfloor M T_{e} /\left(\widetilde{N T}_{c}+\widetilde{D T}_{c}\right)\right\rfloor$.

For a sake of simplicity let us introduce the matrix $V=A A^{\mathrm{H}}$ and $W=B B^{\mathrm{H}}$ of which the components are expressed as follows

$$
V_{l^{\prime} l}=\sum_{i=1}^{\tilde{K} N} A^{*}{ }_{l i} A_{l^{\prime} i} \quad W_{l l^{\prime}}=\sum_{j=1}^{K N} B_{l j} B^{*}{ }_{l^{\prime} j}
$$

for all $l=1, \ldots, M$ and $l^{\prime}=1, \ldots, M$.

By replacing these expressions in Eq. (5) we get

$$
\left\|A^{\mathrm{H}} B\right\|_{F}^{2}=\sum_{l=1}^{M} \sum_{l^{\prime}=1}^{M} V_{l^{\prime} l} W_{l l^{\prime}}
$$

A first application of the Cauchy-Schwartz inequality to the sum of index $l^{\prime}$ in Eq. (6) writes

$$
\sum_{l=1}^{M} \sum_{l^{\prime}=1}^{M} V_{l^{\prime} l} W_{l l^{\prime}} \leq \sum_{l=1}^{M}\left[\left(\sum_{l^{\prime}=1}^{M}\left|V_{l^{\prime} l}\right|^{2}\right)^{\frac{1}{2}}\left(\sum_{l^{\prime}=1}^{M}\left|W_{l l^{\prime}}\right|^{2}\right)^{\frac{1}{2}}\right]
$$

We consider the following notations

$$
\begin{aligned}
& \text { - } v_{l}=\left(\sum_{l^{\prime}=1}^{M}\left|V_{l^{\prime} l}\right|^{2}\right)^{\frac{1}{2}} \\
& \text { - } w_{l}=\left(\sum_{l^{\prime}=1}^{M}\left|W_{l l^{\prime}}\right|^{2}\right)^{\frac{1}{2}}
\end{aligned}
$$

We replace these terms in the RHS term of Eq. (7) and we apply the Cauchy-Schwartz inequality to the sum of index $l$

$$
\sum_{l=1}^{M} v_{l} w_{l} \leq\left(\sum_{l=1}^{M}\left|v_{l}\right|^{2}\right)^{\frac{1}{2}}\left(\sum_{l=1}^{M}\left|w_{l}\right|^{2}\right)^{\frac{1}{2}}
$$

By replacing $v_{l}$ and $w_{l}$ by their respective expressions we deduce that

$$
\sum_{l=1}^{L}\left|v_{l}\right|^{2}=\left\|A^{\mathrm{H}} A\right\|_{F}^{2} \text { and } \sum_{l=1}^{L}\left|w_{l}\right|^{2}=\left\|B^{\mathrm{H}} B\right\|_{F}^{2}
$$


Finally, using Eq. (7) and Eq. (8), it is straightforward to deduce the result of Eq. (3).

Proposition 2: The equality $\mathbf{F}_{\boldsymbol{\theta}} \mathbf{F}_{\boldsymbol{\theta}}^{\mathrm{H}}=\mathbf{F}_{\boldsymbol{\theta}_{\mathbf{0}}} \mathbf{F}_{\boldsymbol{\theta}_{\mathbf{0}}}^{\mathrm{H}}$ holds if and only if $\boldsymbol{\theta}=\boldsymbol{\theta}_{\mathbf{0}}$

Before giving the proof of Proposition 2, it will be worthwhile to study the behavior of the proposed algorithm in a time missynchronization context which occurs if the beginning of the receive signal does not match with the beginning of an OFDM symbol. Indeed, this problem can distort the effectiveness of the proposed cost function. In this context, the receive signal can be expressed as follows

$$
\tilde{y}(m)=y(m-\tau)
$$

where $y$ is the perfectly synchronized signal of Eq. (1).

To deal with this impairment, the proposed algorithm has to be expanded by introducing the new matrix model $\mathbf{F}_{\tilde{\theta}_{0}}$ where $\tilde{\boldsymbol{\theta}}_{0}=\left[N T_{c}, D T_{c}, N, \tau\right]$. This matrix is obtained by the truncation of the first $\left\lfloor\tau / T_{e}\right\rfloor$ rows of $\mathbf{F}_{\boldsymbol{\theta}_{0}}$.

Using this new model matrix, we propose to maximize the following cost function according to the new vector of the unknown parameters $\tilde{\boldsymbol{\theta}}=\left[\widetilde{N T}_{c}, \widetilde{D T}_{c}, \tilde{N}, \tilde{\tau}\right]$ :

$$
J(\tilde{\boldsymbol{\theta}})=\frac{\mathbb{E}\left\{\left\|\mathbf{F}_{\tilde{\boldsymbol{\theta}}}^{\mathrm{H}} \mathbf{y}\right\|^{2}\right\}}{\left\|\mathbf{F}_{\tilde{\boldsymbol{\theta}}} \mathbf{F}_{\tilde{\boldsymbol{\theta}}}^{\mathrm{H}}\right\|_{F}}
$$

Note that Proposition 1 is still verified in this context, moreover, Proposition 2 can be easily expanded as follows:

Proposition 3: The equality $\mathbf{F}_{\tilde{\boldsymbol{\theta}}} \mathbf{F}_{\tilde{\boldsymbol{\theta}}}^{\mathrm{H}}=\mathbf{F}_{\tilde{\theta}_{0}} \mathbf{F}_{\tilde{\boldsymbol{\theta}}_{0}}^{\mathrm{H}}$ is reached if and only if the signal is correctly synchronized and the OFDM parameters are well estimated, i.e., $\tilde{\boldsymbol{\theta}}=\tilde{\boldsymbol{\theta}}_{0}$

The proof of Proposition 2 can be deduced from the proof of Proposition 3 which can be sketched as following.

Proof: We recall that $\mathbf{F}_{\tilde{\theta}_{0}}$ is a block matrix of size $\left(M-\left\lfloor\tau / T_{e}\right\rfloor\right) \times \hat{K} \hat{N}$. The matrix $\mathbf{F}_{\tilde{\boldsymbol{\theta}}_{0}} \mathbf{F}_{\tilde{\boldsymbol{\theta}}_{0}}^{\mathrm{H}}$ is then composed by square blocks matrices as follows:

- the first block is of size $\left\lfloor\left(N T_{c}+D T_{c}\right) / T_{e}\right\rfloor-\left\lfloor\tau / T_{e}\right\rfloor$.

- the size of the other blocks (except the last one) is $\left\lfloor\left(N T_{c}+D T_{c}\right) / T_{e}\right\rfloor$

Consequently, if the equality $\mathbf{F}_{\tilde{\theta}} \mathbf{F}_{\tilde{\boldsymbol{\theta}}}^{\mathrm{H}}=\mathbf{F}_{\tilde{\boldsymbol{\theta}}_{0}} \mathbf{F}_{\tilde{\boldsymbol{\theta}}_{0}}^{\mathrm{H}}$ holds the two sides matrices will have the same block structure. Assuming that $T_{e} \ll \widehat{N T}_{c}+\widetilde{D T}_{c}$, it is straightforward to deduce that $\widehat{N T}_{c}+\widetilde{D T}_{c}=N T_{c}+D T_{c}$ and $\left\lfloor\tilde{\tau} / T_{e}\right\rfloor=\left\lfloor\tau / T_{e}\right\rfloor$ Note that the block structure of $\mathbf{F}_{\tilde{\theta}_{0}} \mathbf{F}_{\tilde{\theta}_{0}}^{\mathrm{H}}$ is sufficient to show the time synchronization condition. Nevertheless, the condition about $N T_{c}$ and $D T_{c}$ require the expression of the equality of each component of $\mathbf{F}_{\tilde{\boldsymbol{\theta}}_{0}} \mathbf{F}_{\tilde{\boldsymbol{\theta}}_{0}}^{\mathrm{H}}$. To express the components of $\mathbf{F}_{\tilde{\boldsymbol{\theta}}_{0}} \mathbf{F}_{\tilde{\boldsymbol{\theta}}_{0}}^{\mathrm{H}}$ let us assume a perfect time sychronization $(\tau=0)$ and an integer number of symbols within the receive signal. Under these assumptions and after some mathematical calculations the components $\mathbf{F}_{\tilde{\boldsymbol{\theta}}_{0}} \mathbf{F}_{\tilde{\boldsymbol{\theta}}_{0}}^{\mathrm{H}}$ can be expressed as follows:

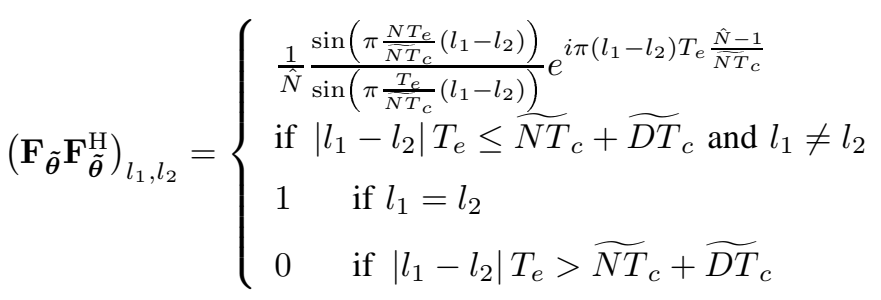

for $l_{1}=1, \ldots, M$ and $l_{2}=1, \ldots, M$.

By assuming that $\mathbf{F}_{\tilde{\theta}} \mathbf{F}_{\tilde{\boldsymbol{\theta}}}^{\mathrm{H}}=\mathbf{F}_{\tilde{\theta}_{0}} \mathbf{F}_{\tilde{\boldsymbol{\theta}}_{0}}^{\mathrm{H}}$, let we consider a nonnull, non-diagonal component with indexes $l_{1}$ and $l_{2}$ such as $\left|l_{1}-l_{2}\right| T_{e} \leq \widetilde{N T}_{c}+\widetilde{D T}_{c}$ and $l_{1} \neq l_{2}$. By performing the phase and modulus equalities we can write:

$$
\left\{\begin{array}{l}
\left(l_{1}-l_{2}\right) T_{e} \frac{N-1}{N T_{c}}=\left(l_{1}-l_{2}\right) T_{e} \frac{N-1}{N T_{c}}+2 k \\
\frac{\sin \left(\pi \frac{N T_{e}}{N T_{c}}\left(l_{1}-l_{2}\right)\right)}{\sin \left(\pi \frac{T_{e}}{N T_{c}}\left(l_{1}-l_{2}\right)\right)}=\frac{\sin \left(\pi \frac{N T_{e}}{N T_{c}}\left(l_{1}-l_{2}\right)\right)}{\sin \left(\pi \frac{T_{e}}{N T_{c}}\left(l_{1}-l_{2}\right)\right)}
\end{array}\right.
$$

where $k \in \mathbb{Z}$.

It is straightforward to show that if these two equalities hold the result can be easily deduced i.e, $\overline{N T}_{c}=N T_{c}$ and $\widetilde{D T}_{c}=$ $D T_{c}$.

\section{IMPACT OF CARRIER FREQUENCY OFFSET}

In a practical context, a frequency offset can alter the received signal. Consequently, the study of this parameter seems to be crucial in order to evaluate its impact on the proposed approach. We consider a non-null component of $\mathbf{F}_{\boldsymbol{\theta}}$ in the model of Eq. (2) with a normalized frequency offset denoted $\delta f$ :

$$
F_{\boldsymbol{\theta}_{\mathbf{0}}}\left(m, k_{m} N+n\right)=\frac{1}{\sqrt{N}} e^{-2 i \pi m T_{e} \frac{n+\delta f}{N T_{c}}} e^{2 i \pi n\left(k_{m}+1\right) \frac{D T_{c}}{N T_{c}}}
$$

where $m=0, \ldots, M-1, k_{m}=0, \ldots, K-1$ and $n=$ $0, \cdots, N-1$.

We propose to extend the proposed algorithm to overcome the effect of the frequency offset. Indeed, we introduce an additional loop to search jointly the unknown OFDM parameters and the frequency offset estimate. Theorem 1 can be modified in order to take into account the unknown carrier offset. Accordingly, we express the following theorem

Theorem 2: By denoting $\boldsymbol{\theta}=\left[N, D T_{c}, N T_{c}, \delta f\right]$ the following inequality still verified:

$$
J(\boldsymbol{\theta}) \leq J\left(\boldsymbol{\theta}_{\mathbf{0}}\right)
$$

and the equality holds if and if $\boldsymbol{\theta}=\boldsymbol{\theta}_{0}$.

The proof of Theorem 2 can be built using Proposition 1 which remains valid and using Proposition 2 which can be extended easily to resolve the problem of the estimation of the carrier offset.

According to this extension, the overall algorithm can be expressed as follows:

- fix the tested value of the intercarrier spacing $\left(1 / \widetilde{N T}_{c}\right)$

- fix the tested value of the guard time duration $\widetilde{D T}$ where $\widetilde{D T}_{c} \in \widetilde{N T}_{c} \times\{1 / 2,1 / 4,1 / 8,1 / 16,1 / 32,0\}$ 


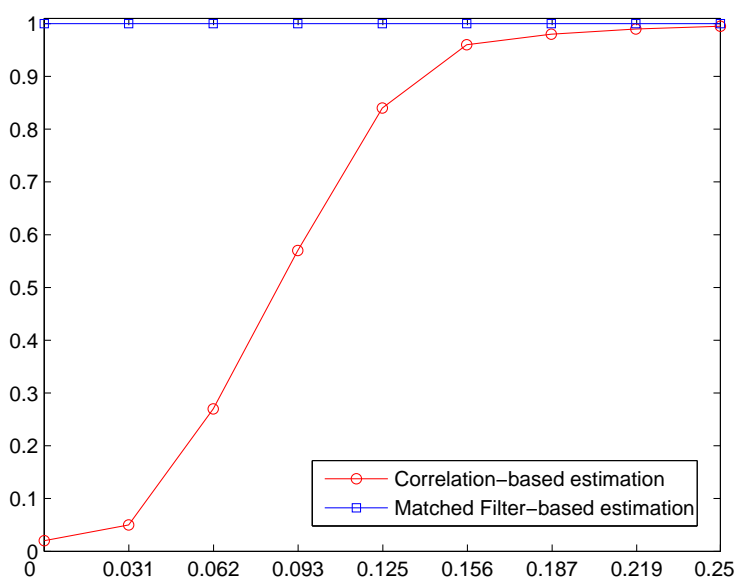

Fig. 1. Correct detection rate vs. $D / N(\mathrm{SNR}=10 \mathrm{~dB})$

- fix the value of the number of carriers $\tilde{N}$

- compensate the carrier offset with a tested value of the normalized frequency offset $\widetilde{\delta f}$ :

$$
z(m)=y(m) e^{2 i \pi m T_{e} \frac{\widetilde{\delta f}}{N T_{c}}}
$$

- drop the first $\left\lfloor\frac{\widetilde{\tau}}{T_{e}}\right\rfloor$ samples of $z$, such as $\tilde{\tau}<\widetilde{N T_{c}}+\widetilde{D T_{c}}$

- evaluate the cost function defined by:

$$
J\left(\widetilde{N T}_{c}, \widetilde{D T}_{c}, \widetilde{N}, \widetilde{\delta f}, \widetilde{\tau}\right)=\frac{\mathbb{E}\left\{\left\|\mathbf{F}_{\boldsymbol{\theta}}^{\mathrm{H}} \mathbf{z}\right\|^{2}\right\}}{\left\|\mathbf{F}_{\boldsymbol{\theta}} \mathbf{F}_{\boldsymbol{\theta}}^{\mathrm{H}}\right\|_{F}}
$$

- update all parameters and restart

- the solution is the vector $\boldsymbol{\theta}=\left[\widetilde{N T}_{c}, \widetilde{D T}_{c}, \widetilde{N}, \widetilde{\delta f}, \widetilde{\tau}\right]$ which maximizes the function $J$.

\section{Simulations}

We propose to evaluate the performance of the proposed algorithm by means of some computer simulations. We recall that the treated problem is the recognition of OFDM based systems in the Cognitive Radio context. As seen in the introduction, an OFDM based system can be identified using the intercarrier spacing. Consequently, we will focus on the estimation of this parameter. Furthermore, as we treat a detection problem we do not need a tight estimation of this parameter but we only need an estimation up to $1 \%$ (cf. Section I) of the right value. The performance of our algorithm will be compared to the state of art technique based on the correlation induced by the cyclic prefix where the following cost function is used to extract the parameter of interest:

$$
\widehat{N T}_{c}=\arg \max _{\widetilde{N T}_{c}}\left\{E\left\{y\left(t+\widetilde{N T}_{c}\right) y^{*}(t)\right\}\right\}
$$

To evaluate these two algorithms, OFDM signals have been generated with $N=64, T_{c}=0.5 \mu \mathrm{s}$ and the sampling time is chosen to be equal to the half of the chip time $T_{e}=T_{c} / 2$.

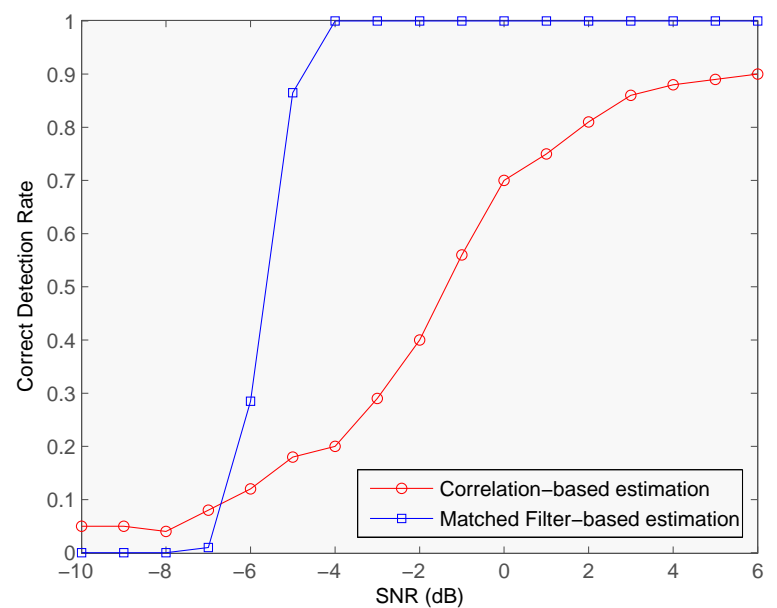

Fig. 2. Correct detection rate vs. $\operatorname{SNR}(D / N=1 / 8)$

The number of available OFDM symbols is 20 . The receive discrete-time signal is written as

$$
y(m)=\sum_{l=0}^{L} h_{l} x(m-l)+b(m)
$$

where $L$ is the length of the channel impulse response and each component of this response is assumed to be Gaussian distributed with zero mean and same variance. Unless otherwise stated, the discrete-time channel dispersion time is fixed to be a quarter of the cyclic prefix duration. $b(m)$ is the noise samples. Unless otherwise stated, Signal-to-Noise Ratio has been fixed to be equal to $10 \mathrm{~dB}$. As performance measure, we consider the percentage where the estimated intercarrier spacing matches with the correct one up to $1 \%$. Consequently, we have considered a grid of values of $\widetilde{N T}_{c}$ of step of $0.3 \mu \mathrm{s}$. For each considered $\widetilde{N T}_{c}$, the cyclic prefix value $\widetilde{D T}_{c}$ takes values in the set $\widetilde{N T}_{c} \times\{1 / 2,1 / 4,1 / 8,1 / 16,1 / 32,0\}$, and $\widetilde{N}$ takes values in the set $\{256,128,64,32\}$. The performance curves have been drawn by averaging the good detection rate over 1000 runs.

First, we propose to evaluate the impact of the ratio between the useful and the cyclic prefix durations on the performance of both algorithms. The measured correct detection rate is showed in Figure 1. As expected, the correlation-based detection fails for small cyclic prefixes because the correlation is strongly decreased. In contrast, the proposed algorithm maintains the same performance whatever the value of the used cyclic prefix. Consequently, the proposed algorithm is more appropriate for the Cognitive Radio which one of several problems is how to treat systems using different $\mathrm{CP}$ lengths. Moreover, notice that within some systems there exist different modes with different values of $\mathrm{CP}$ duration (e.g., WiMax [2]).

In the following, we fix the CP length as $D / N=1 / 8$. We inspect the impact of the Signal to Noise ratio on the performance of each algorithm. Figure 2 shows that the matched 


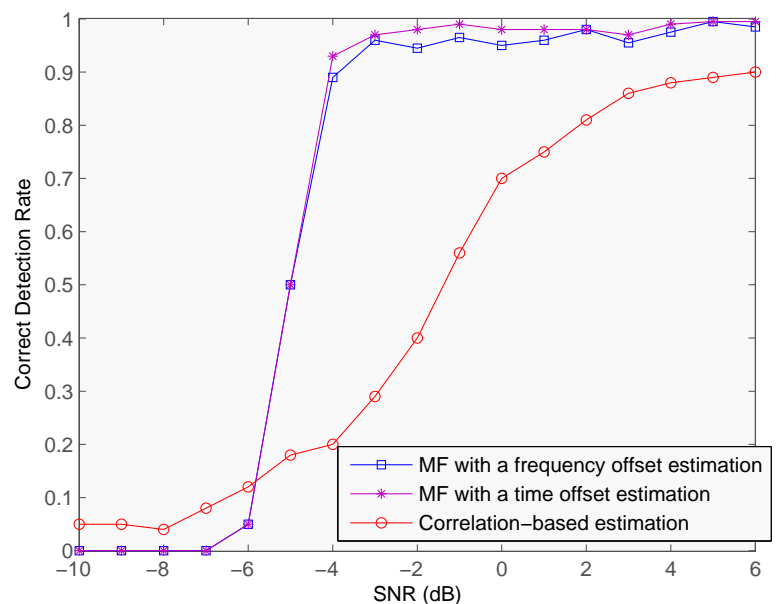

Fig. 3. Correct detection rate vs. SNR including either time offset or frequency offset $(D / N=1 / 8)$

filter based technique outperforms strongly the correlationbased estimation. Indeed, the proposed approaches ensure $100 \%$ of good detections until an SNR of $-4 \mathrm{~dB}$.

Obviously, the perfect frequency and time synchronization are a non realistic hypothesis and thus we need to test the robustness of the proposed approach in a practical situation.

We start by evaluating the effect of the carrier offset. To do that, an OFDM signal has been generated by introducing a random frequency offset. As mentioned in Section IV, an additional loop must be added to perform the joint estimation of both the frequency offset and the OFDM parameters. Figure 3 shows that the proposed approach still work well and resist to the frequency offset impairment. Moreover, it can ensure a good estimation of the carrier offset which allows the receiver to perform data decoding and then extract more information about the received system.

Now we propose to study the effect of the time offset which occurs if the beginning of the received signal does not match with the beginning of an OFDM symbol. Using similar simulations, we show how we can overcome the missynchronization drawbacks. An OFDM signal has been generated and a random number of samples is dropped. The performance of this approach is shown in Figure 3 in contrast with the state of art method. As expected the correlation-based technique is insensitive to a time and a frequency missynchronizations. Nevertheless, it is still less efficient than the modified matched filter-based approach which the performance is as good as the perfect context (cf. Figure 2).

Finally, Figure 4 displays the performance of both methods versus the length of the channel response. The match filter based approach shows a good resistance to multi-paths channel in contrast with the state of art method.

\section{CONCLUSION}

In this paper, we have introduced a new matched filter based algorithm to perform the estimation of the intercarrier spacing

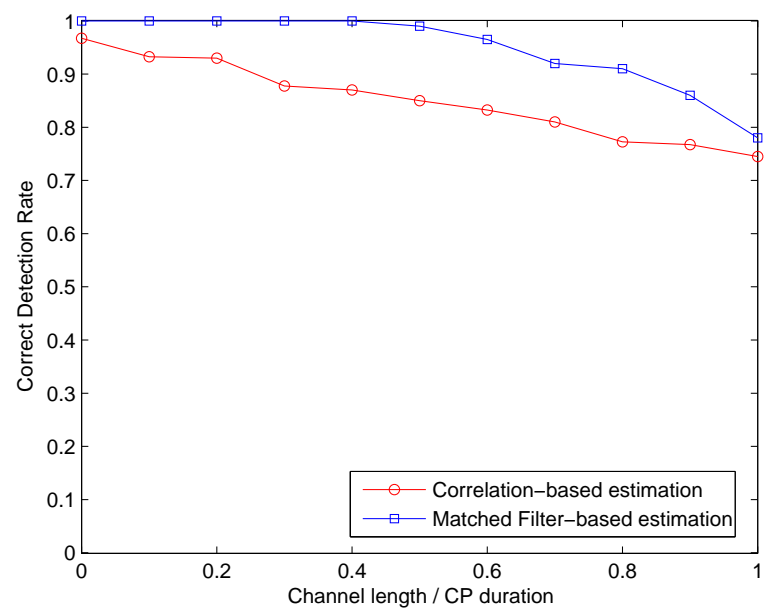

Fig. 4. Correct detection rate vs. the channel response length $(D / N=1 / 8)$

of OFDM modulations. We have proved the effectiveness of the cost function in the context of frequency and time offsets. Obviously, the needed multidimensional optimization induces an additional cost on complexity. Nevertheless, this drawback can be justified by the robustness and the adaptability of the proposed approach. Indeed, we have shown by means of computer simulations that the new method outperforms the state of art approach in terms of signal to noise ratio and the resistance to multi-paths fading channels. Moreover, the proposed algorithm ensures the same performance whatever the used cyclic prefix. Consequently, it is more appropriate to the Cognitive Radio applications and to other totally blind contexts like military applications.

\section{REFERENCES}

[1] J. Mitola, Cognitive Radio : an Integrated Agent architecture for Software Defined Radio, Phd thesis, Royal Institute of Technology (Stockholm, Sweden), 2000.

[2] L. Nuaymi, Wimax: technology for broadband wireless access, John Wiley, 2007.

[3] C. Smith and J. Meyer, 3G wireless with Wimax and Wi-Fi : 802.16 and 802.11, McGraw-Hill, 2005.

[4] ETSI, "Digital videl broadcasting (DVB) framing structure, channel coding and modulation for digital terrestrial television," in ETSI Report, November 2004.

[5] H. Holma and A. Toskala, WCDMA for UMTS: HSDPA evolution and LTE, John Wiley, 2007.

[6] P. Liu, B.-B. Li, Z.-Y. Lu, and F.-K. Gong, "A blind time-parameters estimation scheme for OFDM in multi-path channel," in International Conference on Wireless Communications, Networking and Mobile Computing, Sept. 2005, pp. 242-247.

[7] B. Wang and L. Ge, "Blind identification of OFDM signal in rayleigh channels," in International Conference on Wireless Communications, Networking and Mobile Computing, Dec. 2005, pp. 950-954.

[8] H. Ishii and G.W. Wornell, "OFDM blind parameter identification in cognitive radios," in IEEE International Conference on Personal, Indoor and Mobile Radio Communications, Sept. 2005, pp. 700-705. 